\title{
Retraction Note to: Promoter methylation of DAPK gene may contribute to the pathogenesis of nonsmall cell lung cancer: a meta-analysis
}

\author{
Fan-Fan Li ${ }^{1} \cdot$ Yang Yang $^{1} \cdot$ Xiao-Lei Wang ${ }^{1} \cdot$ Yan-Yan Hong $^{1} \cdot$ Nian-Fei Wang $^{1}$ • \\ Zhen-Dong Chen ${ }^{1}$
}

Published online: 17 August 2015

(C) International Society of Oncology and BioMarkers (ISOBM) 2015

Retraction to: Tumor Biol. (2014) 35:6011-6020

DOI 10.1007/s13277-014-1796-1

The Publisher and Editor retract this article in accordance with the recommendations of the Committee on Publication Ethics (COPE). After a thorough investigation we have strong reason to believe that the peer review process was compromised.

The online version of the original article can be found at http://dx.doi.org/ 10.1007/s13277-014-1796-1.

Fan-Fan Li

1ffdwp@sina.com.cn

$\triangle$ Zhen-Dong Chen

chenzhendong1231@163.com

1 Department of Oncology, The Second Affiliated Hospital of Anhui Medical University, Furong Road No. 678, Hefei Economic and Technological Development Zone, Hefei 230601, People's Republic of China 